\title{
Influence de la dose de soufre élémentaire sur l'activité fermentaire des microbes du rumen dans un système de fermentation semi-continu
}

\author{
S Komisarczuk-Bony, M Carcelen, C Huc
}

INRA, unité de Physio-pathologie du rumen, École nationale vétérinaire, BP 83, 69280 Marcy-l'Etoile, France

\begin{abstract}
Summary - The effects of elemental sulphur level on the activity of rumen microbes in a semicontinuous culture system. The effects of different elemental sulphur levels on rumen fermentation using sulphate sulphur as a control have been studied. Elemental sulphur supply corresponding to $\mathrm{N} /$ $S=2,5$ resulted in a similar level of microbial activity to that of sulphate sulphur at a level of N/S $=10$. No sulphide loss was observed with elemental sulphur, whereas $\approx 50 \%$ of the sulphate sulphur was lost in the form of sulphide.
\end{abstract}

Le soufre (S) élémentaire est généralement reconnu comme la forme la moins disponible pour les micro-organismes du rumen in vitro (Kahlon et al, 1975). Cependant, in vivo, après une période d'adaptation suffisante, cette forme présenterait les mêmes aptitudes que le sulfate à satisfaire les microbes du rumen (Fron et al, 1990). Le but de cette expérimentation est de déterminer dans un système de culture semicontinu quelle quantité de soufre élément on doit fournir aux micro-organismes pour leur permettre d'optimiser leur activité fermentaire en utilisant le soufre sous forme de sulfate de sodium comme référence.

Matériel et méthodes - Quatre fermenteurs de type Rusitec reçoivent journellement 13 $\mathrm{g}$ de cellulose pure et $3 \mathrm{~g}$ d'amidon soluble. L'azote est perfusé en continu sous forme non protéique (urée) à raison de $300 \mathrm{mg} / \mathrm{j}$. Le soufre est apporté journellement en dose unique. Le fermenteur témoin reçoit $30 \mathrm{mg}$ de soufre sulfate $(\mathrm{N} / \mathrm{S}=10)$ et les 3 autres respectivement : $30(\mathrm{~N} / \mathrm{S}=10), 60(\mathrm{~N} / \mathrm{S}=5)$ et $120 \mathrm{mg}(\mathrm{N} / \mathrm{S}=$ $2,5)$ de soufre élément. Après une semaine d'adaptation, on mesure journellement la production d'acides gras à courte chaîne (AGCC), de gaz de fermentation et la dégradation de la cellulose. La protéosynthèse dans la phase liquide est estimée par la différence $\mathrm{N}$ total - $\mathrm{N}$ $\mathrm{NH}_{3}$. Les sulfures (dissous et gazeux) sont dosés par une électrode spécifique (Tacussel XS260). Les fermenteurs sont manipulés journellement, comme décrit par Czerkawski et Breckenridge (1977). Pour chaque paramètre étudié, la moyenne des fermenteurs recevant le soufre élémentaire est comparée à la moyenne du fermenteur témoin recevant le soufre sulfate par un test de Student $(n=11)$.

Résultats et discussion - Les résultats rapportés sur la figure 1 montrent qu'avec le soufre élément, aux doses d'apport $\mathrm{N} / \mathrm{S}=10$ et 5 , l'ensemble des processus fermentaires est significativement réduit par rapport à l'apport témoin de soufre sulfate. Cependant, lorsque la fourniture soufrée atteint une valeur 4 fois supérieure à celle du soufre sulfate, aucune différence significative n'est plus observée entre les 2 formes de soufre. De plus, dans le fermenteur recevant le soufre élément au rapport $N / S=2,5$, les valeurs de pourcentages molaires des différents AGCC (acétate : $50,2 \% \pm 2,9$, propionate : $43,6 \% \pm 3$ et butyrate : $4,7 \% \pm 0,2)$, du rapport $\mathrm{CO}_{2} / \mathrm{CH}_{4}(4,57 \pm 0,27)$ et du rendement de la protéosynthèse microbienne $(25,12$ $\mathrm{gN} / \mathrm{kgMOF} \pm 1,8)$ ne sont pas significativement différentes $(P \leq 0,05)$ du témoin recevant du soufre sulfate au rapport $\mathrm{N} / \mathrm{S}=10$. 
Lorsqu'il est distribué sous forme de sulfate, environ $15 \%$ du soufre introduit sont perdus pour la flore sous forme de sulfures gazeux et $15 \%$ restent non incorporés en $24 \mathrm{~h}$ et sont retrouvés sous forme de sulfures dissous dans les effluents. La vitesse de réduction du sulfate en sulfure est très rapide et la concentration de sulfures atteint un maximum environ $4 \mathrm{~h}$ après introduction dans le fermenteur (fig 2). Par contre, avec le soufre élément, la production de sulfure est faible, et seulement $1,5 \%$ du $S$ introduit se retrouve sous forme de sulfures gazeux.

En conclusion, cette expérimentation montre que le soufre élément, à condition d'être distribué en quantité 3 à 4 fois supérieure au soufre sulfate, permet de satisfaire l'ensemble des besoins des micro- organismes du rumen. Compte tenu de sa faible solubilité et de sa lente dissimilation sous forme de sulfures, le soufre élément devrait présenter l'avantage d'une disponibilité plus régulière dans le temps que le soufre sulfate. Des expérimentations ultérieures chercheront à mettre en évidence l'aptitude comparée de ces 2 formes d'apport soufré pour la supplémentation de régimes naturels à base de fourrages pauvres caractérisés par de faibles vitesses de dégradation dans le rumen.

Czerkawski JW, Breckenridge G (1977) $B r J r$ Nutr 38, 371-384

Fron MJ, Boling JA, Bush LP, Dawon KA (1990) $J$ Anim Sci 68, 543-552

Kahion TS, Meiske JC, Goodrich RD (1975) J Anim Sci 41, 1147-1153

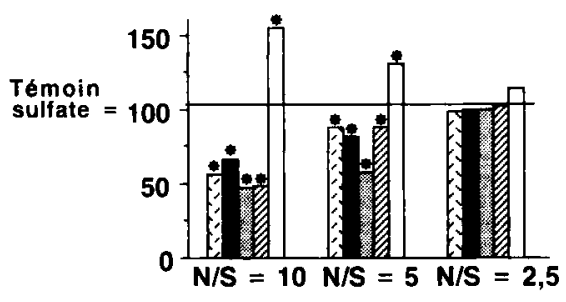

Fig 1. Influence de la dose de soufre élémentaire sur les fermentations du rumen $(\%$ du témoin sulfate). * La différence par rapport au témoin est significative à $P \leq 0,05$.

[₹] AGCC; 2 N microbien; \#. Gaz totaux;

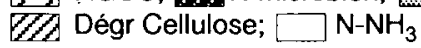

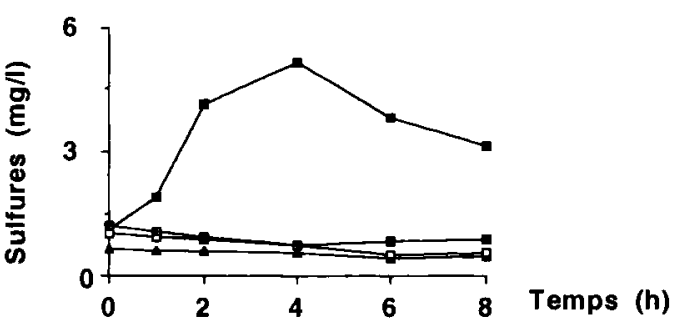

Fig 2. Influence de la dose de soufre élémentaire sur la production de sulfures dans les fermenteurs.

$\longrightarrow$ Témoin SO4; - $\mathrm{A}-\mathrm{N} / \mathrm{S}=10 ; \square \square-\mathrm{N} /$

$S=5 ;-N-N / S=2,5$. 